\title{
Utility of the sore throat pain model in a multiple-dose assessment of the acute analgesic flurbiprofen: a randomized controlled study
}

\author{
Bernard Schachtel ${ }^{1,2^{*}}$, Sue Aspley ${ }^{3}$, Adrian Shephard ${ }^{3}$, Timothy Shea ${ }^{3}$, Gary Smith ${ }^{3}$ and Emily Schachtel ${ }^{2}$
}

\begin{abstract}
Background: The sore throat pain model has been conducted by different clinical investigators to demonstrate the efficacy of acute analgesic drugs in single-dose randomized clinical trials. The model used here was designed to study the multiple-dose safety and efficacy of lozenges containing flurbiprofen at $8.75 \mathrm{mg}$.

Methods: Adults $(n=198)$ with moderate or severe acute sore throat and findings of pharyngitis on a TonsilloPharyngitis Assessment (TPA) were randomly assigned to use either flurbiprofen $8.75 \mathrm{mg}$ lozenges $(n=101)$ or matching placebo lozenges $(n=97)$ under double-blind conditions. Patients sucked one lozenge every three to six hours as needed, up to five lozenges per day, and rated symptoms on 100-mm scales: the Sore Throat Pain Intensity Scale (STPIS), the Difficulty Swallowing Scale (DSS), and the Swollen Throat Scale (SwoTS).
\end{abstract}

Results: Reductions in pain (lasting for three hours) and in difficulty swallowing and throat swelling (for four hours) were observed after a single dose of the flurbiprofen $8.75 \mathrm{mg}$ lozenge $(P<0.05$ compared with placebo). After using multiple doses over 24 hours, flurbiprofen-treated patients experienced a 59\% greater reduction in throat pain, $45 \%$ less difficulty swallowing, and $44 \%$ less throat swelling than placebo-treated patients (all $P<0.01)$. There were no serious adverse events.

Conclusions: Utilizing the sore throat pain model with multiple doses over 24 hours, flurbiprofen $8.75 \mathrm{mg}$ lozenges were shown to be an effective, well-tolerated treatment for sore throat pain. Other pharmacologic actions (reduced difficulty swallowing and reduced throat swelling) and overall patient satisfaction from the flurbiprofen lozenges were also demonstrated in this multiple-dose implementation of the sore throat pain model.

Trial registration: This trial was registered with ClinicalTrials.gov, registration number: NCT01048866, registration date: January 13, 2010.

Keywords: Acute pain, Flurbiprofen, Nonsteroidal anti-inflammatory agent, Pharyngitis, Sore throat

\section{Background}

The sore throat pain model has been used to demonstrate the efficacy of acute analgesic drugs and dosages of these drugs in single-dose randomized placebo-controlled studies [1-4]. As for most acute pain conditions, patients with painful pharyngitis due to upper respiratory tract infection (URTI) often require repeated treatment beyond one or two doses, especially on the first day of treatment, until

\footnotetext{
* Correspondence: bschachtel@srcresearch.net

'Department of Epidemiology \& Public Health, Yale University School of Medicine, 60 College Street, New Haven, CT 06520-8034, USA

${ }^{2}$ Schachtel Research Company, Inc, 4300 So. US Highway One, Suite 203, Jupiter, FL 33477, USA

Full list of author information is available at the end of the article
}

the URTI resolves [5,6]. However, the vis medicatrix naturae of sore throat [7-9], earache [10], and other types of acute pain $[11,12]$ makes it difficult to distinguish an active analgesic from placebo when treatments are re-tested over time, even over 24 hours.

Recognizing this investigational challenge in examining multiple-dose efficacy in patients with an acute, selflimited infectious disease, we employed four specific principles of research architecture in order to tighten the design of this multiple-dose trial. First, to avoid confusion with other expressions of URTI that can also cause a sore throat (such as laryngitis) and might respond differently to treatment, we enrolled only patients with sore throat due 
to pharyngitis. Homogeneity of diagnosis was confirmed on the Tonsillo-Pharyngitis Assessment (TPA), an index of distinct clinical features of pharyngitis $[1,13,14]$. Measurements on the TPA also helped confirm that patients in the treatment groups had the same severity of the physical condition causing sore throat.

Second, to fully characterize the many expressions of throat dis-ease which the patients described, we sought to evaluate other dimensions of throat pain in addition to the evaluative quality of 'sore' throat (pain intensity). We asked the patients to evaluate two other prominent throat-related symptoms which they also complained of, the sensation of a 'swollen throat', a sensory quality commonly reported by patients with sore throat, as well as 'difficulty swallowing', a throat function (dysphagia, distinctly different from odynophagia) [4,5,15-18].

Third, because patients with acute sore throat require treatment mostly during the early course of disease, we sought to enroll patients within three days of their first throat symptom (even a mild sore throat, such as a 'throat tickle'). Thus, we would be more likely to observe patients who provided assessments of sore throat throughout the first 24 hours of the clinical trial. Also, to help assure that symptom severity would be more likely to persist for at least 24 hours and efficacy could be assessed over this treatment period, patients were eligible only if they reported at least a moderate severity of sore throat pain, swollen throat, and difficulty swallowing at baseline.

Previous studies have demonstrated the efficacy and safety of a single dose of a lozenge containing the nonsteroidal anti-inflammatory drug flurbiprofen in patients with sore throat $[7-9,19,20]$, including a single-dose study on different dosages of flurbiprofen lozenge [21]. Here we present the results of a clinical trial utilizing the sore throat pain model to examine the efficacy and safety of multiple doses of a lozenge containing flurbiprofen $8.75 \mathrm{mg}$, with a focus on the initial 24 hours of treatment.

\section{Methods}

\section{Study design}

This was a randomized (ratio 1:1), double-blind, placebocontrolled, multiple-dose, parallel-group study. The first subject was enrolled in November 2009 and the last subject completed in March 2011. The study was conducted in accordance with the International Conference on Harmonization Good Clinical Practice and the ethical principles contained within the Declaration of Helsinki (South Africa, 1996). The study also complied with the Code of Federal Regulations (CFR) of the United States Food and Drug Administration (FDA) and the United States Good Clinical Practice Regulations. The study protocol was reviewed and approved by The New York University School of Medicine's Institutional Review Board, Compass Institutional Review Board and IntegReview Ethical Review Board. The study was registered with the ClinicalTrials.gov registry, registration number: NCT01048866.

\section{Patient selection}

Patients were screened by the study investigators at research sites in the United States after seeking medical care for sore throat, being referred to the trial site, or responding to advertisements about this study. All participants provided written informed consent prior to participation in the study. Adults ( $\geq 18$ years) were included if they had a sore throat of recent onset ( $\leq 3$ days), moderate or severe pain on the Throat Pain Scale (a four-category pain intensity scale), at least one symptom of URTI on the URTI Questionnaire [2,14], objective findings of pharyngeal inflammation $(\geq 5$ on the TPA; Table 1) $[1,13,14]$, and throat symptoms on three patientreported outcome measures: sore throat pain rated more than or equal to $66 \mathrm{~mm}$ on the Sore Throat Pain Intensity Scale (STPIS); difficulty swallowing rated more than or equal to $50 \mathrm{~mm}$ on the Difficulty Swallowing Scale (DSS); and the sensation of a swollen throat rated more than or equal to $33 \mathrm{~mm}$ on the Swollen Throat Scale (SwoTS). See Figure 1 for descriptions of the three symptom rating scales.

Patients were ineligible if they displayed confounding features of URTI: mouth breathing which causes throat drying, coughing which causes throat discomfort, or any disease that could compromise respiratory function. Patients were also ineligible if they had a history of allergic reaction or hypersensitivity to aspirin or other nonsteroidal

Table 1 Tonsillo-Pharyngitis Assessment (TPA)

\begin{tabular}{|c|c|c|c|c|}
\hline Finding & 0 Points & 1 Point & 2 Points & 3 Points \\
\hline Oral temperature & $\leq 98.6^{\circ} \mathrm{F}$ & $98.7-98.9^{\circ} \mathrm{F}$ & $99.0-99.9^{\circ} \mathrm{F}$ & $\geq 100.0^{\circ} \mathrm{F}$ \\
\hline Oropharyngeal color & Normal/pink & Slightly red & Red & Beefy red \\
\hline Size of tonsils & Normal/absent & Slightly enlarged & Moderately enlarged & Much enlarged \\
\hline Number of oropharyngeal enanthems (vesicles, petechiae, or exudates) & None & Few & Several & Many \\
\hline Largest size of anterior cervical lymph nodes & Normal & Slightly enlarged & Moderately enlarged & Much enlarged \\
\hline Number of anterior cervical lymph nodes & Normal & Slightly increased & Moderately increased & Greatly increased \\
\hline Maximum tenderness of some anterior cervical lymph nodes & Not tender & Slightly tender & Moderately tender & Very tender \\
\hline
\end{tabular}




A) The patient was instructed to swallow and:
"Place a line on the 'Sore Throat Scale' that best characterizes the
severity of your sore throat now:'
NO
PAIN PAIN
B) The patient was instructed to swallow and:
"Place a line on the scale that best characterizes how difficult it is to
swallow now:"
NOT
DIFFICULT
C) The patient was instructed to swallow and:
"Place a line on the scale that best characterizes how swollen your
throat feels now:"
NOT
SWOLLEN
Figure 1 Visual analog scales to assess patient-reported outcomes.
The 100-mm visual analog scales used were A) the Sore Throat Pain
Intensity Scale (STPIS) to assess sore throat pain, B) the Difficulty
Swallowing Scale (DSS) to assess difficulty swallowing, and C) the
Swollen Throat Scale (SwoTS) to assess the sensation of a swollen throat.

anti-inflammatory drugs, or any gastrointestinal, hepatic, or renal dysfunction. Patients were excluded if they had used any throat lozenge, throat spray, cough drop, or menthol-containing product within one hour prior to study screening, used any 'cold medication' or immediaterelease analgesic within the previous four hours, used any sustained-release analgesic within the previous 12 hours, taken an antibiotic for an acute disease within 24 hours, or any quinolone antibiotic or inhaled therapy in the previous week.

\section{Study medications}

Under double-blind conditions, patients were randomly allocated to receive sugar-based, flavored flurbiprofen $8.75 \mathrm{mg}$ lozenges or sugar-based, identically flavored placebo lozenges (containing the same vehicle ingredients as the flurbiprofen lozenge). Randomization was achieved using a computer-generated randomization schedule provided by a statistician who was not involved in the analysis of the study. The patients, study investigators, and all other study personnel remained blinded to the treatment allocation after randomization.

Patients were instructed to suck one lozenge (flurbiprofen $8.75 \mathrm{mg}$ or placebo) and were then not permitted to have anything by mouth for the next two hours. Patients were assessed onsite for the first two hours, then discharged with a supply of the same trial lozenges (one lozenge to be used as needed every three to six hours, up to five lozenges in a 24-hour period) and acetaminophen
$650 \mathrm{mg}$ tablets (to be taken as needed every four to six hours if there was inadequate relief from the trial lozenge). No other medications were permitted during this 24-hour period. Over 24 hours (while awake), patients used a diary to record hourly assessments of sore throat pain, swollen throat, and difficulty swallowing, their medication consumption, and any adverse events (AEs). A follow-up visit was conducted for the 24-hour assessments. Over the following six days patients assessed secondary outcomes before and after each self-dosing. At the end of the sevenday observation period patients returned to the site for final assessments and review of AEs and were discharged from the study.

\section{Assessments}

At baseline, all patients were examined for physical findings of tonsillo-pharyngitis, as measured on the TPA (Table 1), and for a global assessment of the severity of pharyngeal inflammation, on the categorical Practitioner's Assessment of Inflammation, or PrAoI (none, mild, moderate, severe). All patients had a throat culture performed to determine the presence of group A betahemolytic streptococcus in the oropharynx. Upon receipt of culture results at 24 to 48 hours, all patients with group A infection received antibiotics and symptomatic patients with group $\mathrm{C}$ streptococcus infection also received antibiotics. All patients continued in the study after the initial 24 hours and were included in the efficacy analyses regardless of their streptococcus infection or antibiotic treatment status.

Previously validated $100-\mathrm{mm}$ visual analog scales (Figure 1) were used to record the symptoms of sore throat pain intensity, difficulty swallowing, and the sensation of a swollen throat $[1,4,18,22,23]$. Patients assessed throat symptoms on the STPIS, DSS, and SwoTS at baseline, one hour, and two hours after the first dose, then every hour for the remainder of the first 24-hour study period (when awake), and again at pretreatment, and one and two hours after each dose taken over days two to seven.

Sore throat relief was also measured at one hour and two hours after the first dose on a validated scale, the Sore Throat Relief Rating Scale (STRRS), a six-category scale ranging from 'no relief' to 'complete relief' $[15,23]$.

At the 24-hour visit patients were asked to rate their satisfaction or dissatisfaction with the study medication by completing the Patient Satisfaction Scale, a sevencategory scale ranging from 'extremely dissatisfied' to 'extremely satisfied' [24].

\section{Statistical analyses}

The primary endpoint of this study was the time-weighted summed difference in pain intensity on the STPIS over 24 hours after the first dose of study medication (SPID24). 
Based on a previous study utilizing this endpoint [21], and assuming a $20 \%$ effect size [7-9] with $80 \%$ power, 200 patients were needed for this study (100 patients on each treatment).

Efficacy was calculated using least square (LS) means, and analysis of variance (ANOVA) was used to compare flurbiprofen $8.75 \mathrm{mg}$ and placebo groups, with treatment and site included as a fixed effect and the relevant baseline (baseline STPIS, DSS, or SwoTS score) included as a covariate. The time-weighted summed differences in DSS and SwoTS over 24 hours were similarly evaluated. The time-weighted summed differences for all three outcomes were assessed over two hours after each dosing over days two to seven. If a patient used rescue medication for pain, all subsequent STPIS, DSS, and SwoTS scores in the 24-hour interval were assigned the baseline value (baseline observation carried forward, BOCF). Missing scores for time-weighted summed differences from baseline were imputed using linear interpolation, assuming the time of the missing assessment to be the nominal time since the first dose, in order to give a more reliable approximation of the AUC from the nonmissing data. Two-sided statistical tests were performed with significance determined by reference to the $5 \%$ significance level.

Wilcoxon rank sum tests were performed to analyze the data obtained for the pharyngeal inflammation category (on the PrAoI), duration of sore throat, size of tonsils (on the TPA), sore throat relief, and patient satisfaction. The chi-square test was performed to analyze the sex and $\mathrm{AE}$ data, and two-sided statistical t-tests were used to analyze the other parameters assessed at baseline.
To determine the efficacy of a single dose of study medication as measured on the STPIS, DSS, and SwoTS $[1,4,5,22,23]$, the absolute change from baseline was calculated over the six hours after the initial single dose of study medication. The odds of achieving at least a $20 \%$ reduction in symptom severity (on the STPIS, DSS, or SwoTS) with flurbiprofen $8.75 \mathrm{mg}$ compared with placebo was assessed using logistic regression, after adjusting for site effects. Treatment effect was also analyzed by determining the cumulative percentage of responders (patients who reported at least a 20\% reduction from baseline in STPIS score) over the initial six hours. These data were used to calculate the absolute risk difference (ARD) between the proportion of responders for the two treatment groups and to calculate the number needed to treat (NNT; 1/ARD). For patients who used additional study or rescue medication in the initial six-hour treatment period, all subsequent changes in STPIS, DSS, and SwoTS scores over six hours were set to zero according to the BOCF convention. All statistical analyses were performed using SAS version 9.2, SAS Institute Inc., Cary, NC, USA.

\section{Results}

\section{Patient enrollment, disposition, and demographics}

Of the 336 patients screened, 198 patients were randomized to receive either flurbiprofen $8.75 \mathrm{mg}(\mathrm{n}=101)$ or placebo $(n=97)$ lozenges (Figure 2). One patient (in the placebo treatment group) withdrew consent and did not record any information between 2 and 24 hours post initial dose, eight patients (four in each treatment group) discontinued between days two and seven; 189 patients

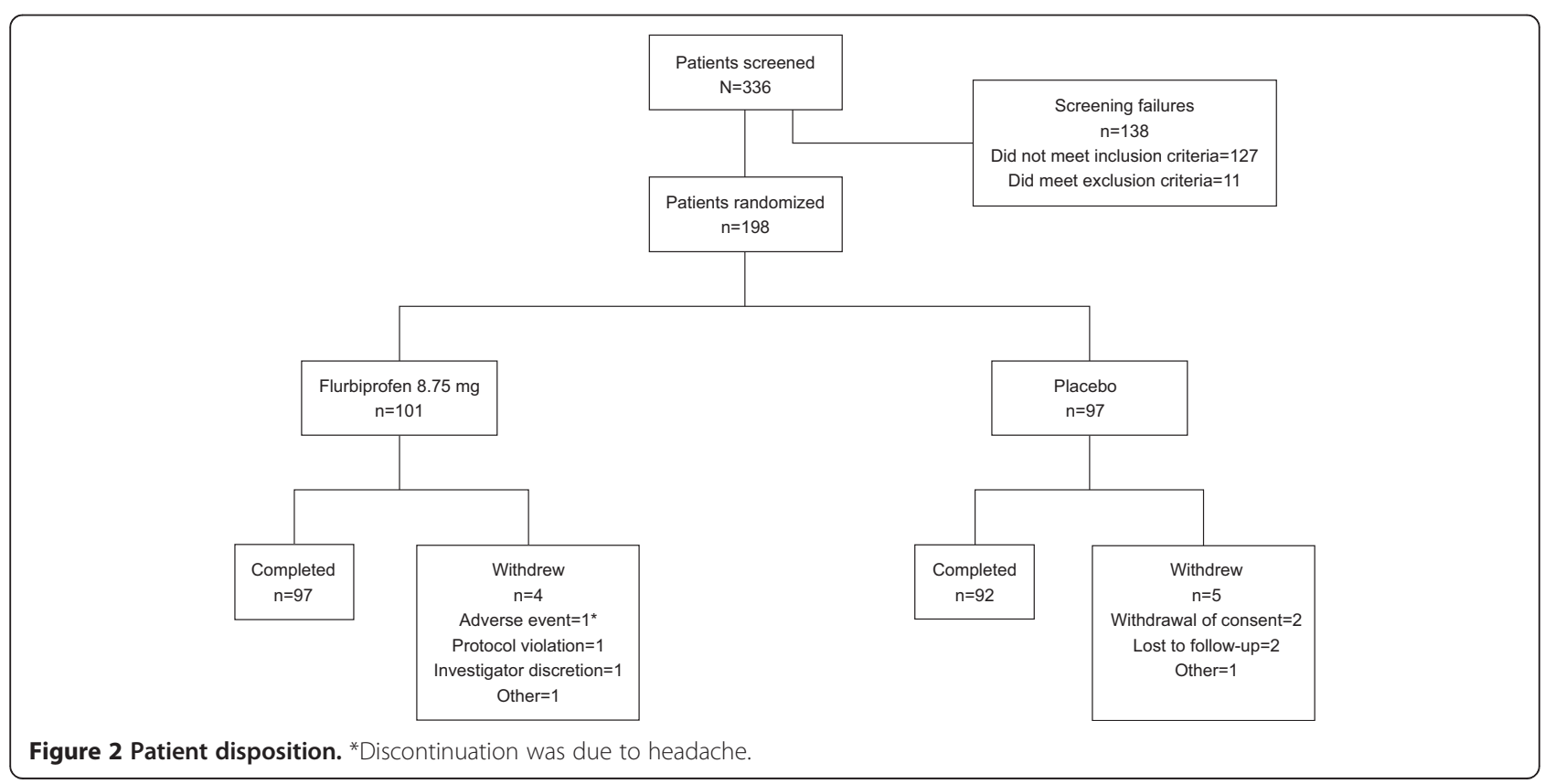


completed the study. Patients in both treatment groups had comparable baseline clinical features $(P>0.1$ for differences between groups). Only $4.1 \%$ of the patients had group A beta-hemolytic streptococcal pharyngitis, with no difference between the treatment groups $(3.0 \%$ in the flurbiprofen $8.75 \mathrm{mg}$ group, $5.2 \%$ in the placebo group; $P>0.1$ ), and most patients had evidence of moderate or severe pharyngeal inflammation (Table 2). The pretreatment severity of all three pharyngeal symptoms (on the STPIS, DSS, and SwoTS) was also comparable (all $P>0.1$ ). Most patients had moderate pain at baseline $(69.3 \%$ of patients in the flurbiprofen $8.75 \mathrm{mg}$ treatment group and $66 \%$ in the placebo group; $P>0.1$ ) (Table 2).

\section{Efficacy}

On average, patients used 4.7 lozenges (standard deviation (SD) 0.96, range 2 to 8 ) over the initial 24-hour treatment period. For the study's primary endpoint (SPID24), patients in the flurbiprofen $8.75 \mathrm{mg}$ group reported a 59\% greater reduction in pain than patients taking placebo (difference of $-196.6 \mathrm{~mm} \times \mathrm{h}$; $95 \%$ confidence interval (CI) -321.0 to $-72.2 ; P<0.01$; Figure $3 \mathrm{~A}$ ). Patients taking flurbiprofen $8.75 \mathrm{mg}$ lozenges also experienced a $45 \%$ greater relief of difficulty swallowing (difference of -179.7 ; 95\% CI -305.7 to $-53.8 ; P<0.01$; Figure $3 \mathrm{~B}$ ) and a $44 \%$ greater reduction of swollen throat than patients taking placebo lozenges over the 24-hour period (difference of -168.4 ; $95 \%$ CI -293.7 to $-43.1 ; P<0.01$; Figure 3C). A greater percentage $(53.6 \%)$ of patients in the flurbiprofen $8.75 \mathrm{mg}$ treatment group reported they were 'satisfied, 'very satisfied', or 'extremely satisfied' with treatment than patients in the placebo group $(38.5 \% ; P<0.05)$.

When the effects of a single dose were analyzed, a statistically significant reduction in sore throat pain intensity was observed for patients treated with flurbiprofen $8.75 \mathrm{mg}$ lozenge compared with placebo up to three hours post-dose $(P<0.01)$. Reductions in absolute DSS and SwoTS levels were also observed at each hourly assessment up to and including four hours post-dose for patients in the flurbiprofen $8.75 \mathrm{mg}$ treatment group compared with placebo (all $P<0.05$ ) (Figure $4 \mathrm{~A}-\mathrm{C}$ ). Significantly greater percentages of flurbiprofen-treated patients reported at least a $20 \%$ reduction in sore throat pain intensity than patients in the placebo treatment group (61.4 and $37.1 \%$ respectively; $P<0.001$ ), in difficulty swallowing (57.4 and $36.1 \%$ respectively; $P<0.01$ ), and in swollen throat (63.4 and $40.2 \% ; P<0.01)$. Using a cumulative responder analysis of patients achieving at least 20\% reduction in sore throat pain intensity (Figure 5), the ARD between flurbiprofen and placebo was 0.22 (NNT 4.5) at one hour post-dose and 0.24 (NNT 4.1) at six hours post-dose.

Sore throat relief was significantly greater for patients who used the flurbiprofen $8.75 \mathrm{mg}$ lozenge than placebo
Table 2 Patient demographics and baseline characteristics

\begin{tabular}{lccc}
\hline Characteristic & $\begin{array}{c}\text { Flurbiprofen } \\
\mathbf{8 . 7 5} \mathbf{~ m g ~ ( n = 1 0 1 )}\end{array}$ & $\begin{array}{c}\text { Placebo } \\
(\mathbf{n}=\mathbf{9 7})\end{array}$ & $\begin{array}{c}\text { Overall } \\
(\mathbf{n}=\mathbf{1 9 8})\end{array}$ \\
\hline Sex, male, $\mathrm{n}(\%)$ & $40(39.6)$ & $39(40.2)$ & $79(39.9)$ \\
$P$ value & & & 0.9311
\end{tabular}

Age (years)

Mean \pm SD

Range

$33.5 \pm 11.0$

$34.2 \pm 11.2 \quad 33.9 \pm 11.1$

$P$ value

$18-61 \quad 18-58 \quad 18-61$

Duration of sore throat, $\mathrm{n}(\%)$

$\begin{array}{lccc}1 \text { day } & 24(23.8) & 19(19.6) & 43(21.7) \\ 2 \text { days } & 39(38.6) & 31(32.0) & 70(35.4) \\ 3 \text { days } & 37(36.6) & 43(44.3) & 80(40.4) \\ 4 \text { days } & 0(0.0) & 3(3.1) & 3(1.5) \\ 5 \text { days } & 1(1.0) & 1(1.0) & 2(1.0) \\ P \text { value } & & & 0.1250\end{array}$

Pain severity, n (\%)

Moderate

$70(69.3) \quad 64(66.0) \quad 134(67.7)$

Severe

$31(30.7)$

33 (34.0)

$64(32.3)$

$P$ value

Mean TPA score \pm SD

$8.0 \pm 2.26$

$8.1 \pm 2.58$

0.6168

$P$ value

0.7630

TPA - size of tonsils, n (\%)

Normal or absent

$27(26.7) \quad 25(25.8) \quad 52(26.3)$

Slightly enlarged

$42(41.6) \quad 37(38.1) \quad 79(39.9)$

Moderately enlarged

$27(26.7) \quad 31(32.0) \quad 58(29.3)$

Much enlarged

$5(5.0)$

$4(4.1)$

$9(4.5)$

$P$ value

0.6635

PrAol, n (\%)

No inflammation

Mild inflammation

$0(0.0)$

$1(1.1)$

$1(0.5)$

Moderate inflammation

42 (41.6)

$37(38.5)$

$79(40.1)$

Severe inflammation

$52(51.5)$

$53(55.2)$

$105(53.3)$

$P$ value

Mean STPIS score \pm SD

$P$ value

$\begin{array}{lll}7(6.9) & 5(5.2) \quad 12(6.1)\end{array}$

0.9550

Mean DSS score \pm SD

$79.1 \pm 8.4$

$79.1 \pm 8.2$

0.9972

$P$ value

$77.9 \pm 10.6$

$78.2 \pm 10.4 \quad 78.0 \pm 10.5$

0.8173

Mean SwoTS score \pm SD

$76.0 \pm 12.9$

$76.0 \pm 12.8 \quad 76.0 \pm 12.8$

$P$ value

0.9739

DSS, Difficulty Swallowing Scale; PrAol, Practitioner's Assessment of Inflammation; SD, standard deviation; STPIS, Sore Throat Pain Intensity Scale; SwoTS, Swollen Throat Scale; TPA, Tonsillo-Pharyngitis Assessment.

at one hour (mean (SD) scores 1.8 (1.22) and 1.2 (1.15), respectively; $P<0.01$ ) and two hours (mean $(\mathrm{SD})$ scores 2.0 (1.23) and 1.3 (1.24); $P<0.0001$ ), the two time points relief was directly measured (on the STRRS). 

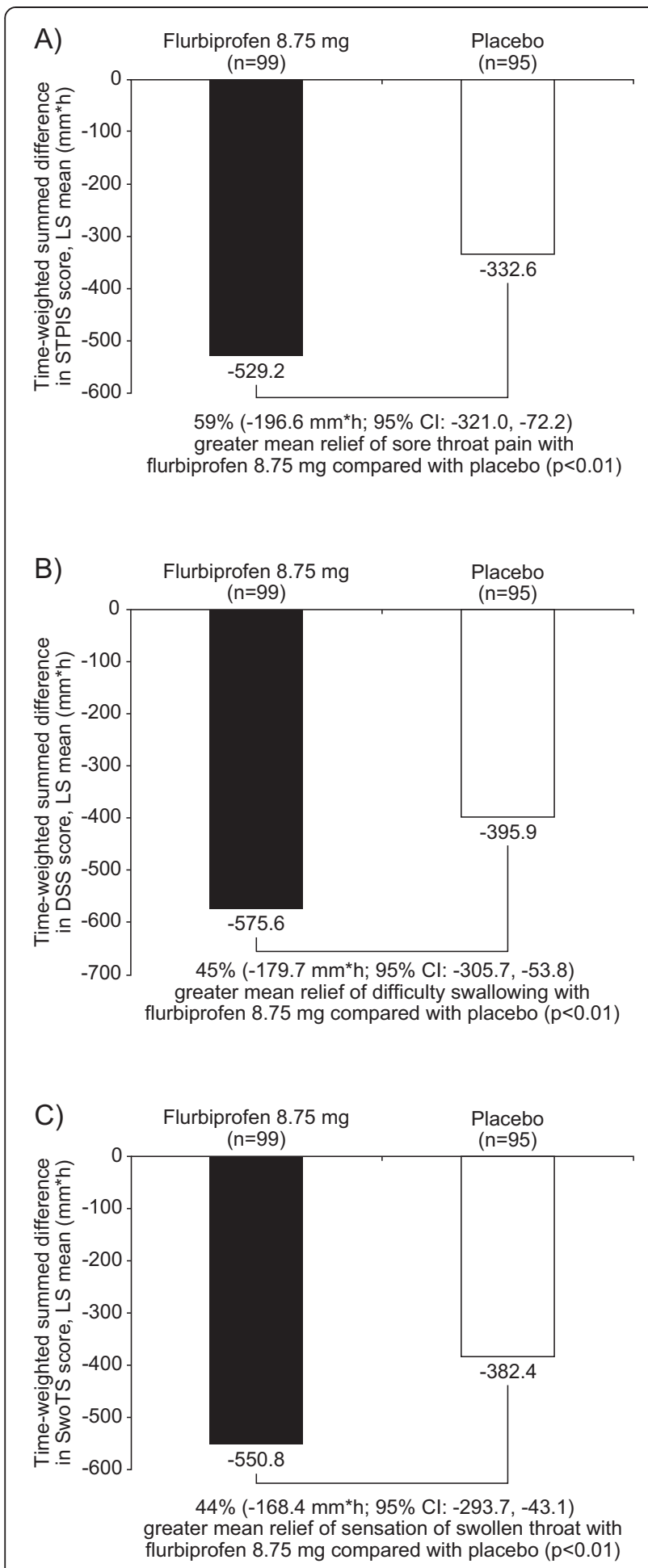

Figure 3 Efficacy of multiple doses of flurbiprofen $8.75 \mathrm{mg}$ or placebo lozenges over $\mathbf{2 4}$ hours. ANOVA was used to compare flurbiprofen $8.75 \mathrm{mg}$ and placebo groups for the time-weighted summed differences in LS mean scores over 24 hours for $\mathbf{A}$ ) sore throat pain (on the STPIS), B) difficulty swallowing (on the DSS), and C) swollen throat (on the SwoTS). ANOVA, analysis of variance; Cl, confidence interval; DSS, Difficulty Swallowing Scale; h, hour; LS, least square; STPIS, Sore Throat Pain Intensity Scale; SwoTS, Swollen Throat Scale.
Patients taking flurbiprofen $8.75 \mathrm{mg}$ lozenges over days two to seven experienced a $38.0 \%$ greater relief of sore throat pain (difference of -8.2 ; $95 \% \mathrm{CI}-17.1$ to 0.7 ; $P=0.07$ ), a $36.9 \%$ greater improvement in difficulty swallowing (difference of $-7.5 ; 95 \% \mathrm{CI}-16.2$ to $1.3 ; P=0.09$ ), and a $45.0 \%$ greater relief of swollen throat compared with patients taking placebo lozenges (difference of -8.1 ; $95 \% \mathrm{CI}-16.8$ to $0.6 ; P=0.07$ ).

\section{Safety}

The percentage of patients who reported any $\mathrm{AE}$ in the first 24 hours of the study (when most lozenges were consumed) was similar for both treatment groups (25.7\% in the flurbiprofen $8.75 \mathrm{mg}$ group, $19.6 \%$ in the placebo group; $P>0.1$ ). Most AEs were related to URTI symptoms (headache and throat irritation). Severe AEs were reported by $1.0 \%$ of patients in both treatment groups $(P>0.1)$; the incidence of gastrointestinal AEs related to study treatment was also similar between groups (occurring in 3.0 and $1.0 \%$ of patients in the flurbiprofen $8.75 \mathrm{mg}$ and placebo treatment groups, respectively; $P>0.1$. Over seven days, the proportion of patients reporting any $\mathrm{AE}$ remained similar between the treatment groups (33.7\% in the flurbiprofen $8.75 \mathrm{mg}$ group, $28.9 \%$ in the placebo group; $P>0.1$ ), as was the incidence of treatment-related gastrointestinal AEs (3.0\% in the flurbiprofen $8.75 \mathrm{mg}$ group, $2.1 \%$ in the placebo group; $P>0.1$ ).

\section{Discussion}

To evaluate the multiple-dose effects of an analgesiccontaining lozenge in patients with sore throat, a selflimited inflammatory pain condition, this randomized, double-blind, placebo-controlled study required strict adherence to basic principles of clinical trial design and methodology. By tightening the admission requirements (specificity of diagnosis, acute onset, and severity of throat symptoms), eliminating confounding clinical features, using validated rating scales for different throat symptoms (not only pain intensity), and measuring these symptoms over the first 24-hour period when throat symptoms are most prominent and require repeated treatment, this multiple-dose trial was sufficiently sensitive to differentiate an active analgesic drug from placebo. The patients were able to detect distinct differences between the sugar-based, flavored flurbiprofen $8.75 \mathrm{mg}$ lozenges and the sugar-based, identically flavored placebo lozenges for the five patient-reported outcomes (reduction of sore throat pain, difficulty swallowing, swollen throat, sore throat relief, and overall patient satisfaction).

The study was also sensitive to single-dose effects, confirming results of previous studies on a single flurbiprofen lozenge compared with placebo [7-9]. Noteworthy was the demonstration of the onset of significant 
Figure 4 Efficacy of a single flurbiprofen $8.75 \mathrm{mg}$ or placebo lozenge over six hours. ANOVA was used to compare flurbiprofen $8.75 \mathrm{mg}(\mathrm{n}=101)$ and placebo ( $\mathrm{n}=97$ up to two hours, $\mathrm{n}=96$ from three to six hours, as one patient withdrew from the study) for the effects of a single dose on absolute mean change over six hours in A) sore throat pain (on the STPIS), B) difficulty swallowing (on the DSS), and C) swollen throat (on the SwoTS). Error bars represent the 95\% Cls. ${ }^{*} P<0.05,{ }^{* *} P<0.01,{ }^{* * *} P<0.001$ compared with placebo. ANOVA, analysis of variance; $\mathrm{Cl}$, confidence interval; DSS, Difficulty Swallowing Scale; LS, Least square; STPIS, Sore Throat Pain Intensity Scale; SwoTS, Swollen Throat Scale.

differentiation of flurbiprofen $8.75 \mathrm{mg}$ from placebo at the first assessment time point (one hour) on all rating scales, in peak effects at one to three hours, and duration lasting for up to four hours on two of the three scales used to measure hourly effects over six hours. These effects represented at least a $20 \%$ reduction in the severity of each patient-reported outcome for the majority of flurbiprofen-treated patients: the pharmacologic effects of this low dose of flurbiprofen were distinctly differentiated from the demulcent effects of the lozenge base itself.

While this study did achieve its objective of demonstrating pharmacologic activity after multiple doses of study medication, there was one major limitation. Approximately $40 \%$ of the patients reported the onset of throat symptoms in the previous three days (and five patients were inadvertently admitted with onset in the previous four to five days). Therefore, the first 24 hours of the study was actually the fourth, fifth, or sixth day of these patients' symptoms. Acute sore throat is an illness of short duration, with $85 \%$ improving within seven days [6]. This natural course may explain why the differences between active drug and placebo over days two to seven

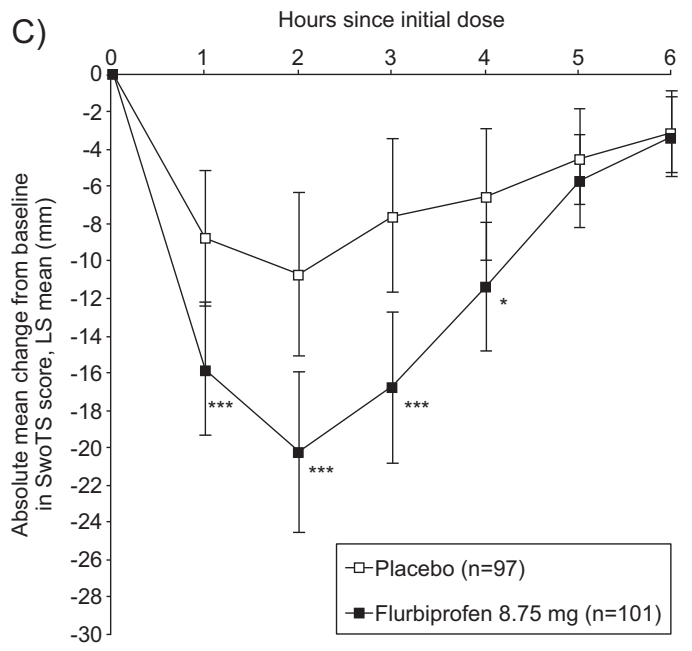

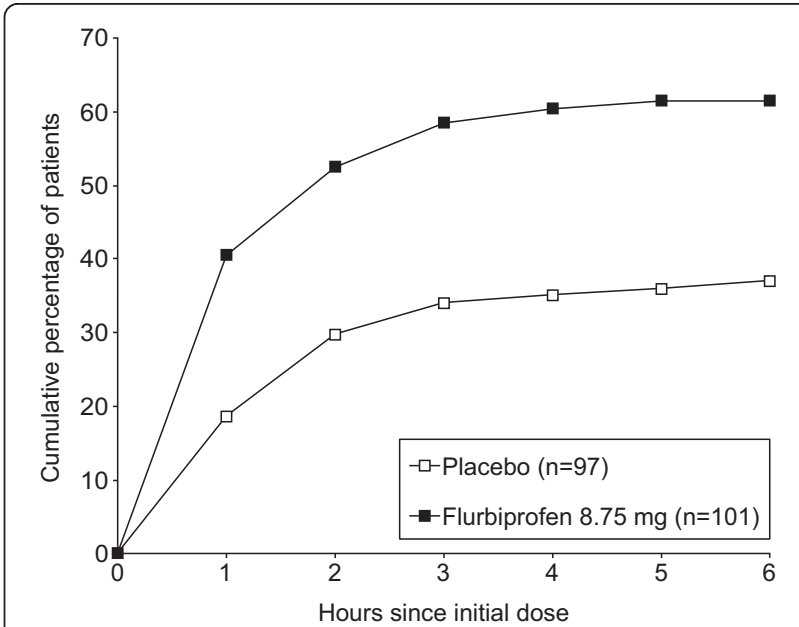

Figure 5 Cumulative percentage of patients achieving at least a $20 \%$ reduction from baseline in Sore Throat Pain Intensity Scale (STPIS) score. 
did not reach statistical significance in the trial. To improve assay sensitivity after the initial stages of sore throat (when symptoms are likely to be worst), future multiple-dose studies on sore throat treatments should restrict recruitment to patients with an onset of only one or two days.

\section{Conclusions}

In this study, using specific design and methods, the sore throat (pharyngitis) pain model demonstrated that multiple doses of flurbiprofen $8.75 \mathrm{mg}$ lozenges are an effective, well-tolerated treatment for patients with sore throat. Efficacy was evident for the patient-reported outcomes of pain intensity, pain relief, and swollen throat as well as throat function (swallowing) and overall patient satisfaction. We therefore conclude that this pain model is a practical and efficient multiple-dose assay for the safety and efficacy of analgesic agents.

\section{Abbreviations}

AE: Adverse event; ANOVA: Analysis of variance; AUC: Area under the curve; BOCF: Baseline observation carried forward; CFR: Code of Federal Regulations; Cl: Confidence interval; DSS: Difficulty Swallowing Scale; FDA: Food and Drug Administration; h: Hour; LS: Least square; PrAol: Practitioner's Assessment of Inflammation; SD: Standard deviation; SPID24: Summed pain intensity difference over 24 hours; STPIS: Sore Throat Pain Intensity Scale; STRRS: Sore Throat Relief Rating Scale; SwoTS: Swollen Throat Scale; TPA: Tonsillo-Pharyngitis Assessment; URTI: Upper respiratory tract infection.

\section{Competing interests}

This study was funded by a research grant from Reckitt Benckiser Healthcare International Ltd., UK. SA, AS, TS, and GS are employees of Reckitt Benckiser Healthcare International Ltd. BS and ES received an investigational grant from Reckitt Benckiser Healthcare International Ltd., UK, to design and conduct this study.

\section{Authors' contributions}

All of the authors have contributed substantially to the study reported in this manuscript and the development of this manuscript: BS, SA, AS, and ES contributed to the study concept and design. GS performed the statistical analyses. BS, TS, and ES were involved in the conduct of the study and acquisition of data. BS, SA, AS, TS, GS, and ES contributed to the interpretation of results. All authors have reviewed and approved this manuscript.

\section{Acknowledgments}

The study was sponsored by Reckitt Benckiser Healthcare International Ltd. Editorial assistance was provided by Elements Communications Ltd. and funded by Reckitt Benckiser Healthcare International Ltd.

\section{Author details}

${ }^{1}$ Department of Epidemiology \& Public Health, Yale University School of Medicine, 60 College Street, New Haven, CT 06520-8034, USA. ${ }^{2}$ Schachtel Research Company, Inc, 4300 So. US Highway One, Suite 203, Jupiter, FL 33477, USA. ${ }^{3}$ Reckitt Benckiser Healthcare International Ltd., 103-105 Bath Road, Slough, Berkshire SL1 3UH, UK.

Received: 20 September 2013 Accepted: 18 June 2014

Published: 3 July 2014

\section{References}

1. Schachtel BP, McCabe D, Berger M, Zhang R, Sanner KM, Savino L, Rizouk J, Schachtel EP: Efficacy of low-dose celecoxib in patients with acute pain. J Pain 2011, 12:756-763.

2. Schachtel BP, Voelker M, Sanner KM, Gagney D, Bey M, Schachtel E, Becka M: Demonstration of the analgesic efficacy and dose-response of acetylsalicylic acid with pseudoephedrine. J Clin Pharmacol 2010, 50:1429-1437.
3. Schachtel BP, Thoden WR: A placebo-controlled model for assaying systemic analgesics in children. Clin Pharmacol Ther 1993, 53:593-601.

4. Schachtel BP, Fillingim JM, Beiter DJ, Lane AC, Schwartz LA: Subjective and objective features of sore throat. Arch Intern Med 1984, 144:497-500.

5. Schachtel BP, Aspley S, Berry P, Muir N, Shephard A, Shea T, Smith G, Schachtel E: Efficacy of a novel (lozenge) delivery of flurbiprofen over 24 hours. J Pain 2012, 13(Suppl):S74.

6. Del Mar CB, Glasziou PP, Spinks AB: Antibiotics for sore throat. Cochrane Database Syst Rev 2006, CD000023.

7. Blagden M, Christian J, Miller K, Charlesworth A: Multidose flurbiprofen $8.75 \mathrm{mg}$ lozenges in the treatment of sore throat: a randomised, doubleblind, placebo-controlled study in UK general practice centres. Int I Clin Pract 2002, 56:95-100.

8. Benrimoj SJ, Langford JH, Christian J, Charlesworth A, Steans A: Efficacy and tolerability of the anti-inflammatory throat lozenge flurbiprofen $8.75 \mathrm{mg}$ in the treatment of sore throat. A randomized double-blind, placebocontrolled study. Clin Drug Invest 2001, 21:183-193.

9. Watson N, Nimmo WS, Christian J, Charlesworth A, Speight J, Miller K: Relief of sore throat with the anti-inflammatory throat lozenge flurbiprofen $8.75 \mathrm{mg}$ : a randomised, double-blind, placebo-controlled study of efficacy and safety. Int I Clin Pract 2000, 54:490-496.

10. Schachtel BP, Thoden WR: Assaying analgesic response in children: a doubleblind, placebo-controlled model involving earache. Pediatr Res 1991, 29:124A.

11. Manvelian G, Daniels S, Gibofsky A: A phase 2 study evaluating the efficacy and safety of a novel, proprietary, nano-formulated, lower dose oral diclofenac. Pain Med 2012, 13:1491-1498.

12. Derry S, Moore RA, McQuay HJ: Single dose oral codeine, as a single agent, for acute postoperative pain in adults. Cochrane Database Syst Rev 2010, (4):CD008099.

13. Aspley S, Schachtel BP, Berry P, Shephard A, Shea T, Smith G, Schachtel E: Flurbiprofen lozenges in patients with a "bad sore throat". J Pain 2013, 14:S59.

14. Schachtel BP, Pan S, Kohles JD, Sanner KM, Schachtel EP, Bey M: Utility and sensitivity of the sore throat pain model: results of a randomized controlled trial on the COX-2 selective inhibitor valdecoxib. $J$ Clin Pharmacol 2007, 47:860-870.

15. Schachtel BP, Fillingim JM, Thoden WR, Lane AC, Baybutt RI: Sore throat pain in the evaluation of mild analgesics. Clin Pharmacol Ther 1988, 44:704-711.

16. Schachtel BP, McCormick CG, Giefer EE: Patient-reported outcomes (PROs) in the pharyngitis pain model. Montreal, Canada: 13th World Congress of the International Association for the Study of Pain; 2010.

17. Aspley S, Schachtel BP, Berry P, Shephard A, Sanner K, Savino L, Rezuke J, Shea T, Smith G: Treatment of odynophagia and dysphagia by flurbiprofen 8.75 mg lozenges. Pain Res Manag 2012, 17:203.

18. Schachtel BP, Fillingim JM, Beiter DJ, Lane AC, Schwartz LA: Rating scales for analgesics in sore throat. Clin Pharmacol Ther 1984, 36:151-156.

19. Schachtel BP, Aspley S, Berry P, Shephard A, Sanner K, Shea T, Smith G, Schachtel E: Chief Complaint: the therapeutogenic stimulus as the primary, individualized endpoint in clinical trials. J Pain 2012, 13:S6.

20. Schachtel BP, Aspley S, Sternberg M, Berry P, Muir N, Sanner K, Rezuke J, Savino L, Shephard A, Shea T, Schachtel E: Onset of demulcent and analgesic activity of flurbiprofen lozenge. Int J Clin Pharm 2012, 34:143-258.

21. Schachtel BP, Homan HD, Gibb IA, Christian J: Demonstration of dose response of flurbiprofen lozenges with the sore throat pain model. Clin Pharmacol Ther 2002, 71:375-380

22. Schachtel BP: Sore throat pain. In The Design of Analgesic Clinical Trials (Advances in Pain Research and Therapy), Volume Volume 18. Edited by Max MB, Portenoy RK, Laska EM. New York: Raven Press; 1991:393-406.

23. Schachtel BP, Fillingim JM, Lane AC, Thoden WR, Baybutt RI: Caffeine as an analgesic adjuvant. A double-blind study comparing aspirin with caffeine to aspirin and placebo in patients with sore throat. Arch Intern Med 1991, 151:733-737.

24. Atkinson MJ, Kumar R, Cappelleri JC, Hass SL: Hierarchical construct validity of the treatment satisfaction questionnaire for medication (TSQM version II) among outpatient pharmacy consumers. Value Health 2005, 8(Suppl 1):S9-S24.

\section{doi:10.1186/1745-6215-15-263}

Cite this article as: Schachtel et al:: Utility of the sore throat pain model in a multiple-dose assessment of the acute analgesic flurbiprofen: a randomized controlled study. Trials 2014 15:263. 